\title{
Formula for Energy Loss in Soft Magnetic Materials and Scaling
}

\author{
K. SokAlski And J. SzczygŁowski \\ Faculty of Electrical Engineering, Technical University of Częstochowa \\ al. Armii Krajowej 17, 42-200 Czȩstochowa, Poland
}

(Received July 17, 2008; revised version September 23, 2008; in final form October 16, 2008)

\begin{abstract}
Basing on scale invariance of considered system an improvement of the Bertotti formula for energy loss in soft magnetic materials has been achieved. Assumptions of the Bertotti theory were discussed and criticized. As an alternative to this theory a new approach basing on the scale invariance of complex systems has been presented. The generalized description of energy loss has been recently postulated by us in the form of the homogeneous function in a general sense which leads to a series expansion for the energy loss. On the basis of measurement data it has been proved that only two first terms of the series are relevant. New measurements of the energy loss in soft magnetic materials have been performed which confirms the scaling theory. The obtained formula enables very simple description of the energy loss in soft magnetic materials, taking into considerations wide ranges of frequency and magnetic induction. The revealed data collapse of energy loss enables comparison of energy losses data taken by different methods. This phenomenon also supplies new criterion for correctness of empirical data.
\end{abstract}

PACS numbers: 75.50.-y, 89.75.Da

\section{Introduction}

Energy loss in soft magnetic material (SMM), subjected to an alternating magnetic field in the range of low and medium frequencies, is the result of eddy currents generated in the material, which is characterized by its specific conductivity. Recently, we have presented an approach to the energy loss in SMM basing on scaling theory [1]. The achieved agreement between experimental data and this theory suggests the extension of performed investigations into the following directions: criticism of the flaws of the current approach to the energy loss in SMM [2-4], discussion of the background of the scaling in SMM, confirmation of the scaling in SMM by the new measurements.

Nowadays, a number of phenomena occurring in different spatial scales in the material is recognized, which can be accounted for the source of induced eddy currents. Eddy currents may be induced on microscale due to the Barkhausen jumps, which results in a theoretical dependence $P_{\text {hys }} \propto f B_{\mathrm{m}}^{\alpha}$, where $f$ is frequency of magnetizing field, $B_{\mathrm{m}}$ is maximum magnetic induction and $\alpha$ is constant, unique for a specific material $[5,2]$, on the intermediate scale i.e. around moving domain walls $P_{\text {exc }} \propto\left(f B_{\mathrm{m}}\right)^{3 / 2}[2,3]$, as well as on macroscale, covering the whole material volume $-P_{\text {clas }} \propto\left(f B_{\mathrm{m}}\right)^{2}[5,2]$.

The contemporary approach to energy loss phenomenon in soft magnetic materials assumes that the total loss $P_{\text {tot }}$ is the sum of described above contributions $[2-4]$ :

$$
P_{\text {tot }}=C_{1} f B_{\mathrm{m}}^{\alpha}+C_{2}\left(f B_{\mathrm{m}}\right)^{2}+C_{3}\left(f B_{\mathrm{m}}\right)^{3 / 2}
$$

where $C_{1}, C_{2}, C_{3}$ are constants. The approach to energy loss, which assumes their additive character, allows for their separation and consequently for the independent analysis of the obtained components in different scales. However, the results obtained in the case of modern materials of amorphous and microcrystalline structure reveal discrepancies between the theoretical and experimental values $[6,7]$. In the seventies of the past century the aforementioned approach to energy loss was criticized as being oversimplified. It was pointed out that the energy loss phenomenon ought to be considered as a whole [6]. This might suggest that the discrepancy between theory and experiment and the separation in the formula (1) are related.

The main aim of this work is to confirm that the total energy loss in soft magnetic materials $P_{\text {tot }}$ obeys the scaling law (9) and to discuss its relation to the Bertotti formula (1). All conclusions of this paper have been derived from measurements of total energy loss, carried out for the ten samples of soft magnetic materials, which exhibit diverse internal structure - from crystalline to amorphous and nanocrystalline. Measurements for the seven samples have been published previously in [1], whereas here we confirm obtained results by the new measurements.

\section{Simplifying assumptions for the Bertotti approach}

In order to find out whether the discrepancy and the separation are correlated one should reconsider ab initio the source of separation in (1). Following this line we focused our attention on the formula for the total eddy current density $\boldsymbol{j}(\boldsymbol{r}, t)$ being a result of the Barkhausen jumps at different random points $\boldsymbol{r}_{i}$ and at different random times $t_{i}$, presented in [2]: 


$$
\boldsymbol{j}(\boldsymbol{r}, t)=\sum_{i=1}^{N} \boldsymbol{j}\left(\boldsymbol{r}, t ; \boldsymbol{r}_{i}, t_{i}\right),
$$

where $\boldsymbol{j}\left(\boldsymbol{r}, t ; \boldsymbol{r}_{i}, t_{i}\right)$ is the contribution to $\boldsymbol{j}(\boldsymbol{r}, t)$ resulting from the Barkhausen jump at $\left(\boldsymbol{r}_{i}, t_{i}\right)$. We find this formula to be extremely rough approximation which does not correlate events at $(\boldsymbol{r}, t)$ and at $\left(\boldsymbol{r}_{i}, t_{i}\right)$ by the electromagnetic wave propagation. It has been argued in [2] that since $\boldsymbol{j}\left(\boldsymbol{r}, t ; \boldsymbol{r}_{i}, t_{i}\right)$ are solutions of the Maxwell equations, which are linear, then the superposition (2) holds.

We prove that $\boldsymbol{j}, \boldsymbol{H}$, and $\boldsymbol{M}$ obey the system of quasi-linear partial differential equations we eliminated the electric field $\boldsymbol{E}$ and the electric induction $\boldsymbol{D}$ from the Maxwell equations. In order to describe the dynamics of $\boldsymbol{M}$ we attach the Brown equation (instead of the Brown equation one can attach any other appropriate model of the magnetization's dynamics). However, the set of equations describing the current density in the soft magnetic material is not linear

$$
\begin{aligned}
& \frac{1}{\gamma} \triangle \boldsymbol{j}-\frac{\mu_{0} \epsilon}{\gamma} \frac{\partial^{2}}{\partial t^{2}} \boldsymbol{j}-\mu_{0} \frac{\partial}{\partial t} \boldsymbol{j}=\nabla \times \frac{\partial}{\partial t} \boldsymbol{M}, \\
& -\triangle \boldsymbol{H}+\mu_{0} \epsilon \frac{\partial^{2}}{\partial t^{2}} \boldsymbol{H}+\epsilon \frac{\partial^{2}}{\partial t^{2}} \boldsymbol{M}=\nabla \times \boldsymbol{j}, \\
& \frac{\partial}{\partial t} \rho_{\mathrm{e}}+\nabla \cdot \boldsymbol{j}=0, \\
& \nabla \cdot\left(\mu_{0} \boldsymbol{H}+\boldsymbol{M}\right)=0, \\
& \frac{\partial}{\partial t} \boldsymbol{M}=g \mu_{0} \boldsymbol{H} \times \boldsymbol{M}+K \triangle \boldsymbol{M} \times \boldsymbol{M},
\end{aligned}
$$

where $\boldsymbol{H}$ - magnetic field strength, $\epsilon$ - dielectric permeability, $\boldsymbol{j}$ - current density vector, $\boldsymbol{M}$ - magnetization vector, $\rho_{\mathrm{e}}$ - charge density, $g$ - electron gyromagnetic ratio, $K$ - exchange integral and $\gamma-$ specific conductivity.

Equation (7) induces the strong nonlinearity in (3) and therefore the system $(3)-(7)$ is not linear and no linear superposition (2) can hold except a regime of weak $\boldsymbol{M}$, where (7) becomes linear. Moreover, it has been said that (2) is valid in the regime of immediate reactions which was equivalent to the approximation of infinite light velocity and contradicted the Maxwell theory.

In order to improve (1) we assume that the considered system is a complex one and it exhibits scale invariance features. Its complexity arises from the nonlinear relation between $\boldsymbol{H}$ and $\boldsymbol{M}$ and makes the system very hard to investigate. Therefore, instead of detailed analysis basing on the Maxwell equations and the Eqs. (3)-(7) resulting from, we propose to apply mathematical tools basing on the global properties of the system. Just the scale invariance is global and can be expressed by the homogeneity of the functions describing the energy loss in SMM.

\section{Soft magnetic material as a complex system}

The concept of scale invariance embodies the idea that a comprehensive description of a material requires an understanding over many time and length scales. It is the pillar of theoretical models for the complex systems which have been tested for many years in critical phenomena and turbulence in inertial range. A heuristic argument could be developed into a possible coherent approach to understanding the ubiquity of scale invariance in a wide range of complex systems. By the scale invariance we mean a hierarchical organization that results as homogeneity in a generalized sense of functions describing properties of considered complex system.

The hierarchical organization in soft magnetic materials concerns the eddy currents distribution. This distribution is determined by the boundary conditions, the domain structure, the domain wall movements and the Barkhausen jumps. These causes generate the wide spectrum of scales, from the sample size up to the lattice constant. We consider this fact as an argument for the assumption that the dissipation of energy in SMM exhibits the scale invariant features.

Therefore, correlations between the currents [8,9] can be expressed with the postulate of the generalized homogeneity of correlation function current density-current density [10]. In a particular case we obtain the scaling relation for the average of $\left|\boldsymbol{j}^{2}\right|$ :

$$
\lambda^{c^{\prime}}\left\langle\left|\boldsymbol{j}(\boldsymbol{r}, t)^{2}\right|\right\rangle=\left\langle\left|\boldsymbol{j}\left(\lambda^{a^{\prime}} \boldsymbol{r}, \lambda^{b^{\prime}} t\right)^{2}\right|\right\rangle, \quad \forall \lambda>0,
$$

where $a^{\prime}, b^{\prime}, c^{\prime}$ are scaling exponents. The quantity $P_{\text {tot }} \approx$ $\left\langle\left|\boldsymbol{j}(\boldsymbol{r}, t)^{2}\right|\right\rangle$ denotes energy loss in the case of soft magnetic materials. The symbol $\langle\ldots\rangle$ denotes averaging with respect to time and over the whole sample volume. On the basis of Eq. (8), it can be assumed that the total energy loss in soft magnetic materials $P_{\text {tot }}=F\left(f, B_{\mathrm{m}}\right)$ is given by a generalized homogeneous function

$$
\begin{aligned}
P_{\text {tot }} & =F\left(f, B_{\mathrm{m}}\right), \quad \lambda^{c} P_{\mathrm{tot}}=F\left(\lambda^{a} f, \lambda^{b} B_{\mathrm{m}}\right), \\
\forall \lambda>0, &
\end{aligned}
$$

where $P_{\text {tot }}$ - total energy loss, $B_{\mathrm{m}}$ - maximal magnetic induction, $f$ - frequency of magnetizing field, $a, b, c-$ scaling exponents.

\section{Scaling of energy loss in soft magnetic materials}

Assumption, given by Eq. (9), immediately leads to the following formula for energy loss [1]:

$$
\begin{aligned}
P_{\mathrm{tot}} & \approx B_{\mathrm{m}}^{\beta}\left[\Gamma^{(1)} \frac{f}{B_{\mathrm{m}}^{\alpha}}+\Gamma^{(2)}\left(\frac{f}{B_{\mathrm{m}}^{\alpha}}\right)^{2}\right. \\
+ & \left.\Gamma^{(3)}\left(\frac{f}{B_{\mathrm{m}}^{\alpha}}\right)^{3}+\Gamma^{(4)}\left(\frac{f}{B_{\mathrm{m}}^{\alpha}}\right)^{4}+\ldots\right],
\end{aligned}
$$

where $\alpha, \beta$ are related to exponents of Eq. (9):

$$
\beta=\frac{c}{b} \quad \text { and } \quad \alpha=\frac{a}{b},
$$

and $\Gamma^{(n)}$ 's are the arbitrary coefficients.

Relation (10) is the generalized homogeneous function, therefore its every term describes energy loss phenomenon in a wide range of spatial scales. At this point of considerations we are able to indicate an internal contradiction in the Bertotti formula (1). In general, the contemporary used relation (1) is not the generalized homogeneous function (for $\alpha \neq 1$ ), whereas its every 
term is homogeneous. On the other hand, the accessible experimental data [1] as well as results of the new measurements presented here are strong arguments for the assumption that $P_{\text {tot }}\left(f, B_{\mathrm{m}}\right)$ is a uniform function in generalized sense. Therefore, the relation (1) does not describe a self-similar system, as it results from the empirical investigations. Taking into consideration that all terms in (1) are homogeneous in general meaning, their sum is not homogeneous, it should be stated that this relation is not self-consistent.

The phenomenological approach to energy dissipation in soft magnetic materials, proposed in [1], reveal that every term of relation (10) includes effective interactions of the other terms, and consequently their total sum is also the generalized homogeneous function, for any values of exponents $\alpha$ and $\beta$. It allows for their estimation without violation of the homogeneity principle. Thereby, considerations presented in further part of the paper are based on relation (10).

\subsection{Experimental data}

Measurements of total energy loss $P_{\text {tot }}$ were carried out for the three samples of different classes of soft magnetic materials, which exhibit diverse internal structures and magnetic properties:

- crystalline material - non-oriented electrotechnical steel sheets $3 \% \mathrm{Si}-\mathrm{Fe}$,

- amorphous material $\mathrm{Fe}_{78} \mathrm{Si}_{13} \mathrm{~B}_{9}$,

- Iron-nickel alloy $77 \% \mathrm{Ni}-\mathrm{Fe}$.

Samples made of non-oriented electrical steel had the shape of strip, whereas samples made of the remaining magnetic materials had the shape of cylinder.

The measurements of total energy losses were carried out as a function of maximum induction $B_{\mathrm{m}}$, at fixed values of frequency $f$. The induction $B_{\mathrm{m}}$ was changed in the range from $0.1 \mathrm{~T}$ up to $0.7 \mathrm{~T}$ for iron-nickel alloys and up to $1.8 \mathrm{~T}$ for grain-oriented steel, step $0.1 \mathrm{~T}$. The range of frequency changes was from 10 to $400 \mathrm{~Hz}$. Thereby, for each magnetic material the set of curves of total energy losses $P_{\text {tot }}$ vs. maximum induction $B_{\mathrm{m}}$ and frequency $f$ was obtained. Next, the energy loss measurements were carried out following to the norm IEC60404-2. The norm's requirements concern the magnetic properties' measurements of the electrical steel strip and sheet by means of an Epstein frame [11] and consist in the followings:

1. general principles of ac measurements,

2. procedure for the measurement of the specific total loss,

3. procedure for the determination of the peak value of magnetic polarization, rms value of magnetic field strength, peak value of magnetic field strength and specific apparent power,
4. general principles of dc measurements,

5. procedure for the dc measurement of the magnetic polarization.

The standards for magnetic measurements of power loss in electrical steels require the wave form of the induced voltage in the secondary coil to be a sinusoid with respect to time, and its form factor must be $1.111 \pm 1 \%$ for the measurement of ac magnetic losses in steels.

In our measurements the shape factor of secondary voltage was equal to $1.111 \pm 0.5 \%$. The extended uncertainty of obtained measurements (repeatability of measurements specified with standard deviation) was approximately $1.5 \%$.

The obtained detailed results in the form of charts $P_{\text {tot }} / B_{\mathrm{m}}^{\beta}$ vs. $f / B_{\mathrm{m}}^{\alpha}$ for three chosen samples were depicted in Figs. 1, 2, and 3. However, measurement results for all samples were depicted in the charts, which illustrate a linear dependence of exponents $\alpha$ and $\beta$ (Fig. 4) and the data collapse (Fig. 5).

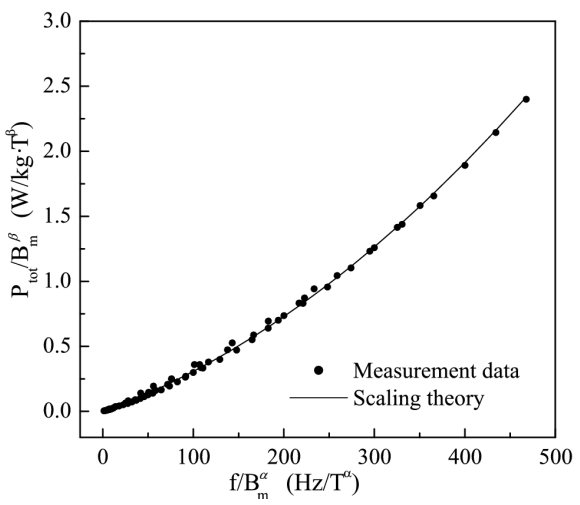

Fig. 1. A comparison of measured (points) and theoretical (solid line) values of scaled total energy loss vs. scaled frequency for amorphous ribbon $\mathrm{Fe}_{78} \mathrm{Si}_{13} \mathrm{~B}_{9}$.

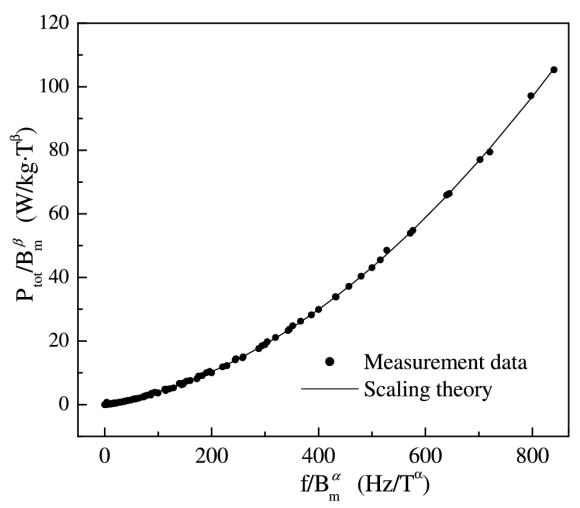

Fig. 2. A comparison of measured (points) and theoretical (solid line) values of scaled total energy loss vs. scaled frequency for non-oriented electrotechnical steel sheets $3 \% \mathrm{Si}-\mathrm{Fe}$. 


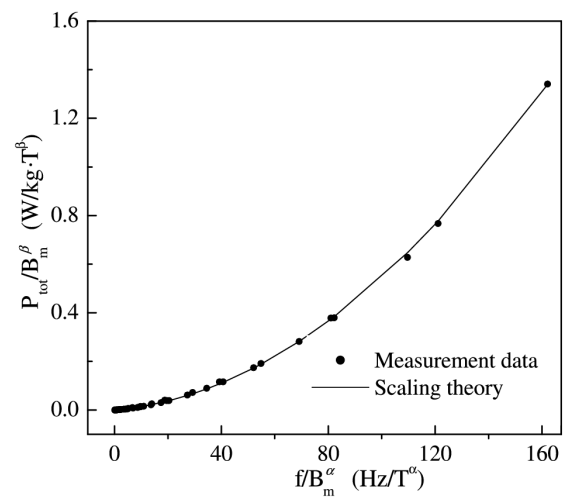

Fig. 3. A comparison of measured (points) and theoretical (solid line) values of scaled total energy loss vs. scaled frequency iron-nickel alloys $77 \% \mathrm{Ni}-\mathrm{Fe}$.

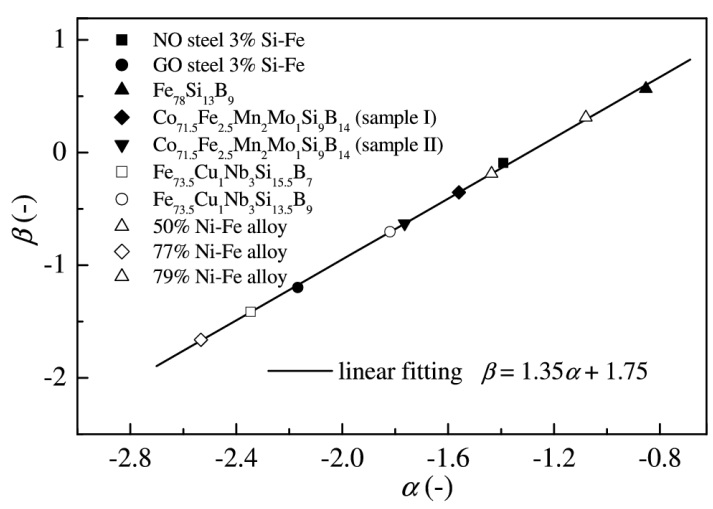

Fig. 4. A phenomenological relation between the exponents $\alpha$ and $\beta$ (points - measurement data, solid line - linear fit according to Eq. (13)). The three points from Table correspond to measurements presented here, whereas the other seven are taken from [1]. Differences between the samples I and II of $\mathrm{Co}_{71.5} \mathrm{Fe}_{2.5} \mathrm{Mn}_{2} \mathrm{Mo}_{1} \mathrm{Si}_{9} \mathrm{~B}_{14}$ result from the variability of the technological parameters.

\subsection{Parameter estimation of the generalized homogeneous function}

In order to estimate the values of exponents $\alpha$ and $\beta$ as well as the amplitudes $\Gamma^{(n)}$, the minimum chi square method was applied, assuming normal distribution of fitting error. The quantity to be optimized was the sum of squares of differences between the measurement values of total energy loss $P_{\text {tot }}$ and energy loss obtained from Eq. (10), limited to its four first terms. Values of exponents $\alpha, \beta$ and amplitudes $\Gamma^{(1,2)}$ for three chosen magnetic materials (amorphous ribbon $\mathrm{Fe}_{78} \mathrm{Si}_{13} \mathrm{~B}_{9}$, non-oriented electrotechnical steel sheets $3 \% \mathrm{Si}-\mathrm{Fe}$ and iron-nickel alloys $77 \% \mathrm{Ni}-\mathrm{Fe}$ ) are given in Table.

The number of digits after decimal point gives the information about the relative uncertainties of exponent and amplitude estimation. Values of amplitudes $\Gamma^{(3)}$ and

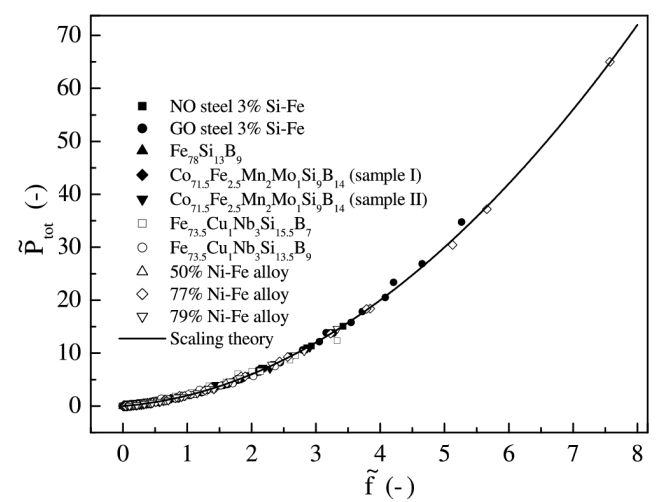

Fig. 5. The data collapse for total energy loss of soft magnetic materials: points - measurements, solid line — theoretical curve according to Eq. (14).

$\Gamma^{(4)}$ are $5 \ldots 15$ orders of magnitude lower, compared to $\Gamma^{(1)}$ and $\Gamma^{(2)}$. Thereby, only amplitudes $\Gamma^{(1)}$ and $\Gamma^{(2)}$ are significant, whereas terms of $\Gamma^{(3)}$ and $\Gamma^{(4)}$ can be omitted, which leads to the following formula:

$$
P_{\mathrm{tot}} \approx B_{\mathrm{m}}^{\beta}\left[\Gamma^{(1)} \frac{f}{B_{\mathrm{m}}^{\alpha}}+\Gamma^{(2)}\left(\frac{f}{B_{\mathrm{m}}^{\alpha}}\right)^{2}\right] .
$$

Figures 1-3 depict measured values of total energy loss for chosen magnetic materials and values of energy loss obtained from the scaling theory and optimization procedures. The scaled measured and theoretical data are presented in the coordinate system $P_{\text {tot }} / B_{\mathrm{m}}^{\beta}=F\left(f / B_{\mathrm{m}}^{\alpha}\right)$.

TABLE

Values of exponents $\alpha, \beta$ and amplitudes $\Gamma^{(1)}, \Gamma^{(2)}$.

\begin{tabular}{c|c|c|c|c}
\hline \hline $\begin{array}{c}\text { Magnetic } \\
\text { materials }\end{array}$ & $\alpha$ & $\beta$ & $\begin{array}{c}\Gamma^{(1)} \\
{\left[\frac{\mathrm{m}^{2}}{\mathrm{~s}^{2}} T^{(\alpha-\beta)}\right]}\end{array}$ & $\begin{array}{c}\Gamma^{(2)} \\
{\left[\frac{\mathrm{m}^{2}}{\mathrm{~s}} T^{(2 \alpha-\beta)}\right]}\end{array}$ \\
\hline $\begin{array}{c}\text { amorphous } \\
\text { ribbon } \\
\mathrm{Fe}_{78} \mathrm{Si}_{13} \mathrm{~B}_{9}\end{array}$ & -0.85 & 0.56 & $2.50 \times 10^{-3}$ & $5.69 \times 10^{-6}$ \\
\hline $\begin{array}{c}\text { non-oriented } \\
\text { steel } \\
3 \% \mathrm{Fe}-\mathrm{Si}\end{array}$ & -1.3923 & -0.09435 & $28.488 \times 10^{-3}$ & $57.9 \times 10^{-6}$ \\
\hline $\begin{array}{c}\text { iron-nickel } \\
\text { alloy } \\
77 \% \mathrm{Ni}-\mathrm{Fe}\end{array}$ & -2.53 & -1.66 & $0.96 \times 10^{-3}$ & $2.25 \times 10^{-6}$ \\
& & &
\end{tabular}

On the basis of measurement data for all analyzed magnetic materials, a relation between the exponents $\alpha$ and $\beta$ (see Fig. 4) in the following form was obtained:

$$
\beta=1.35 \alpha+1.75 \text {. }
$$

Our phenomenological formula (13) is most likely strictly related to the analogous ones acting between various critical exponents and derived from the second order phase transition theory [12]. On the basis of presented results it could be stated that the above relation (13) is universal, whereas the exponents are not. The exponent values may be different due to different dimensions of the samples. The relation (13) enables us to estimate the fea- 
sible values of $\alpha$ and $\beta$. The estimated sets of their values depend on a cut-off of the considered series (10).

Here we present the two results: for the full infinite series (10) and for its two terms approximation (12). Assuming that the considered form for $P_{\text {tot }}$ is not singular in $B_{\mathrm{m}}=0$ we derive the following estimations for $\alpha$ :

$$
\begin{aligned}
& -5<\alpha<0 \text { for }(10), \\
& -5<\alpha<\frac{35}{13} \text { for } \quad(12
\end{aligned}
$$

$\beta$ is determined by (13).

\subsection{Data collapse}

Very small values of $\Gamma^{(3)}$ and $\Gamma^{(4)}$ terms allowed cutting off the expansion (10) above the third order. Because the obtained formula is simple, it was possible to obtain the data collapse by appropriate scaling. Accordingly (12) was transformed to the sample-independent form, which includes the scaled variables $\widetilde{P}_{\text {tot }}$ and $\widetilde{f}$ :

$$
\widetilde{P}_{\text {tot }}=\widetilde{f}+\widetilde{f}^{2}
$$
where

$$
\widetilde{P}_{\text {tot }}=\frac{\Gamma^{(2)}}{\Gamma^{(1)^{2}}} \frac{P_{\mathrm{tot}}}{B_{\mathrm{m}}^{\beta}} \quad \text { and } \quad \tilde{f}=\frac{\Gamma^{(2)}}{\Gamma^{(1)}} \frac{f}{B_{\mathrm{m}}^{\alpha}} .
$$

A chart of all measurement data, scaled according to (15), confirms the data collapse for total energy loss of soft magnetic materials. Figure 5 depicts the revealed data collapse. Figure 6 is an enlarged fragment of Fig. 5 and depicts the distribution of measurement points around theoretical curve given by Eq. (14).

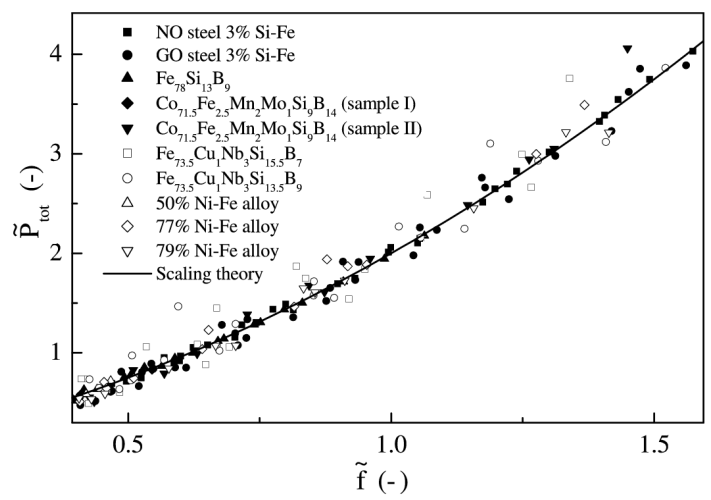

Fig. 6. Enlarged part of Fig. 5 - a distribution of measurements around theoretical curve according to (14).

The revealed data collapse for the energy loss is a definitive confirmation of the scaling hypothesis in reference to the phenomenon of energy loss in soft magnetic materials. The formula (14) describing energy loss is universal. The variable $\widetilde{f}$, which appears in Eq. (14), is the renormalized frequency dressed by magnetic induction and sample geometry. Therefore, the obtained equation is free from the boundary conditions and material-specific quantities, which was possible due to multi-scalability of the analyzed system.

\section{Conclusions}

The presented approach to the energy losses in soft magnetic materials fits very well to accessible empirical data. The first conclusion is that the two-component model (12) is sufficient for the complete description of phenomenon considered here. The second conclusion concerns the universality of the derived results. The formula for the energy losses expressed in dimensionless units (14) as well as the relation between exponents are universal.

This discovery suggests some new practical application of the derived theory. It would be very convenient to characterize the dissipation of energy in the given sample by the three constants: $\Gamma^{(1)}, \Gamma^{(2)}$, and $\alpha$. Knowing their values and applying (14), (15) as well as (13), one can calculate energy loss for an arbitrary values of $B_{\mathrm{m}}$ and $f$. Moreover, reducing $B_{\mathrm{m}}$ and $f$ to $\tilde{f}$ and $P_{\text {tot }}$ to $\widetilde{P}_{\text {tot }}$ one can compare measurements performed on different samples.

In comparison to the Bertotti formula we went back to the two-term expression for the energy loss in SMM. One can ask a question what happened to the term proportional to $f^{3 / 2}$ ? This term results from interactions between eddy currents generated in different points $\left(\boldsymbol{r}_{i}, t_{i}\right)$, whereas in our approach all interactions are taken into account by the very general assumption concerning the scale invariance of SMM which is expressed by (9). In effect we obtain formula (10) depending on $\tilde{f}=f / B_{\mathrm{m}}^{\alpha}$ which we interpret as effective frequency dressed by all types of interactions present in the considered system.

\section{References}

[1] K. Sokalski, J. Szczygłowski, M. Najgebauer, W. Wilczyński, COMPEL 26, 640 (2007).

[2] G. Bertotti, Hysteresis in Magnetism, Academic Press, San Diego 1998.

[3] G. Bertotti, J. Magn. Magn. Mater. 112, 253 (1984).

[4] G. Bertotti, IEEE Trans. Magn. 24, 624 (1988).

[5] R.M. Bozorth, Ferromagnetism, IEEE Magnetic Society, New York 1993.

[6] B.D. Cullity, Introduction to Magnetic Materials, Addison-Wesley Publ. Co., Reading (Mass) 1972.

[7] G.E. Fish, C.-F. Chang, R. Bye, J. Appl. Phys. 64, 5370 (1988).

[8] A.N. Kolmogorov, Dokl. Akad. Nauk SSSR 30, 299 (1941).

[9] A.S. Monin, A.M. Yaglom, Statistical Hydromechanics, Part II, Nauka, Moscow 1976.

[10] G.A. Kuzmin, A.M. Yaglom, Sov. Phys. JEPT 35 620 (1972).

[11] International Electrotechnical Commission, International Standard - $4-60404-2$ CIEC: $1996+\mathrm{A1}$ : 2008, Edition 3.1 2008-06, Magnetic Materials, Part 2.

[12] H.E. Stanley, Introduction to Phase Transitions and Critical Phenomena, Clarendon Press, Oxford 1971. 